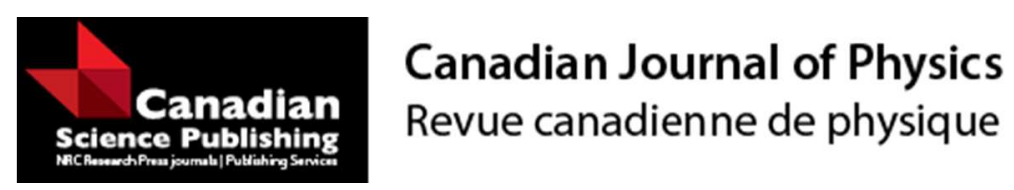

\title{
Dynamics of two dipole-coupled atom system driven by a quantized cavity field
}

\begin{tabular}{|r|l|}
\hline Journal: & Canadian Journal of Physics \\
\hline Manuscript ID & cjp-2016-0762.R2 \\
\hline Manuscript Type: & Article \\
\hline Date Submitted by the Author: & 27-Feb-2017 \\
\hline Complete List of Authors: & $\begin{array}{l}\text { Zhang, Wen-Xuan; Institute of Theoretical Physics } \\
\text { Tan, Lei; Lanzhou Univ, }\end{array}$ \\
\hline Keyword: & $\begin{array}{l}\text { dynamics, entanglement, dipole-dipole interaction, quantized cavity field, } \\
\text { population }\end{array}$ \\
\hline \multicolumn{2}{|l}{} \\
\hline
\end{tabular}

SCHOLARONE ${ }^{\text {IM }}$

Manuscripts 


\title{
Dynamics of two dipole-coupled atom system driven by a quantized cavity field
}

\author{
Wen-Xuan Zhang ${ }^{1}$ and Lei $\operatorname{Tan}^{1,2, *}$ \\ ${ }^{1}$ Institute of Theoretical Physics, Lanzhou University, Lanzhou 730000, China \\ ${ }^{2}$ Key Laboratory for Magnetism and Magnetic materials of the \\ Ministry of Education, Lanzhou University, Lanzhou 730000, China
}

(Dated: March 13, 2017)

\begin{abstract}
Based on the master-equation approach, we investigate the dynamics of two dipole-coupled atoms by examining the atomic population and entanglement evolution. We show that the population decays rapidly and the entanglement is inhabited when the pump field is weak, while for the strongly pumped case, the evolution of population and entanglement is similar with one driven in the classical field. Furthermore, the evolution of the population can be controlled by the diploe-diploe interaction and detunings.
\end{abstract}

PACS numbers: 42.50.Pq 37.30.+i, 42.60.Da, 05.30.Jp

\section{INTRODUCTION}

Cavity quantum electrodynamics (CQED), as a platform for investigating the interaction between photons and atoms, has been widely studied in different fields with the development of experimental techniques. A wealth of phenomena have been predicted and realized successfully. The system of two-atom interaction with a cavity field is a straightforward extension model of the fundamental model of Jaynes-Cummings that describes the interaction between a single atomic dipole transition and a single mode of the radiation field sustained by a high-finesse resonator in the optical frequency domain [13]. With the development of atom trapping and cooling techniques, two atoms can be trapped at distances on the order of a resonant wavelength, which makes the applicability of the dipole-dipole interaction (DDI) between two atoms questionable[4-9]. In recent years, the twoatom system has attracted considerable attention both in theory and experiments, and it has been extensively investigated with and without considering the effects of the DDI. These include population revival [10], squeezing revival[11], transmission spectrum $[12,13]$ and photon statistics [14-18]. Furthermore, the quantum correlation $[19,20]$ and entanglement dynamics $[21-26]$ of the two-atom system have also been discussed by different research groups. Most recently, the collective radiation of two atoms in a cavity coherently driven by an external laser has been investigated [27].

In view of the rich and varied applications mentioned above, the system of two-atoms has become an interesting subject of study. Most of the work has been carried out in the classically driven cases. The atoms were usually assumed to be trapped in a prescribed potential formed by stationary fields independent of the atoms, which can be described by a c-number function. This requires intense light, far detuned from any atomic tran-

\footnotetext{
*Electronic address: tanlei@lzu.edu.cn
}

sition. Of course, this assumption no longer holds if the optical field, which generates the optical potential, is enhanced by a high-finesse optical cavity field. In addition, the quantized cavity field and the atoms are also mutually interacting [28-31]. That is, the cavity field acts as a probe field to couple the atoms, while the atoms, in turn, induce a shift in the index of refraction, altering the cavity field. As the field adjusts accordingly, the field causes the atoms to react, resulting in the nonlinear atom-field interplay. This is very important in several aspects [31], including cavity cooling[32], optical bistability[33] and transmitted spectra [13]. To obtain further information on the two-atom system, the cavity field itself is required to quantize, and the dynamical quantity, which depends on the atomic distribution, should be considered. In the present paper, we consider the effect of a quantized field on the two-atom system, taking into account the dipoledipole interaction based on a coupled equation that includes the quantized cavity field. The dynamics of the atoms and the time evolution of entanglement are investigated. A comparison with the results obtained in a classical field driven case is also made.

This paper is organized as follows. In Sec.II, the model of two two-level atoms with DDI coupled with a quantized cavity field will be established, and the dissipation of the whole system will be introduced. In Sec.III, we will discuss the time evolution of the atomic population using different parameters and study the effect of quantized field and DDI on the population. Sec.IV is devoted to a discussion of the entanglement of atoms and the photon count rate in the given conditions. Finally, we present our conclusions in Sec.V.

\section{MODEL}

We consider a system that involves two identical twolevel atoms with excited states $\left|e_{i}\right\rangle$ and ground states $\left|g_{i}\right\rangle(i=i$ th atom $)$ driven by a single-mode quantized cavity field. Here, the atoms interact with each other through DDI induced by photon exchange. We also assume that the system is pumped by a coherent laser filled 


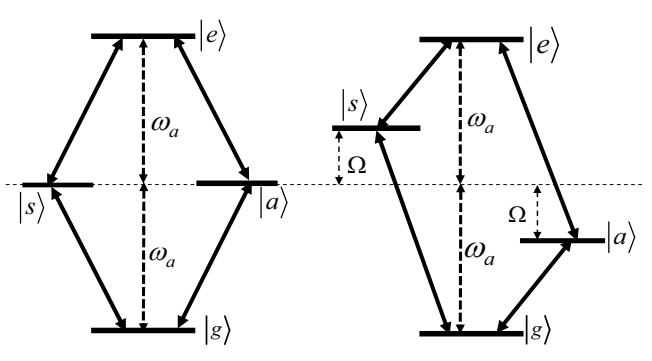

Figure 1: (Color online) Schematic diagram of level energy for the two atoms is illustrated. The intermediate states $|s\rangle$ and $|a\rangle$ occur shift in energy level through the effect of DDI between the atoms. $\Omega$ is the coupling strength of DDI between atoms, and $\omega_{a}$ represents the frequency of the quantized cavity field.

with an effective amplitude $\eta$ with optical frequency $\omega_{p}$. The Hamiltonian of this system in the rotating wave approximation can be written as $(\hbar=1)[2]$

$$
\begin{aligned}
H= & -\Delta_{a} \sum_{i=1}^{2} S_{i}^{z}-\Delta_{c} a^{\dagger} a+\sum_{i=1}^{2} g\left(a^{\dagger} S_{i}^{-}+a S_{i}^{+}\right) \\
& +\Omega\left(S_{1}^{+} S_{2}^{-}+S_{1}^{-} S_{2}^{+}\right)+\eta\left(a^{\dagger}+a\right),
\end{aligned}
$$

where $\Delta_{a}=\omega_{p}-\omega_{a}, \Delta_{c}=\omega_{p}-\omega_{c} . \omega_{a}$ and $\omega_{c}$ are the resonance frequencies of atoms and the quantized cavity field. $a^{\dagger}$ and $a$ represent the creation and annihilation operators of the quantized cavity field. $S_{i}^{z}$ represents the energy operator of $i t h$ the atom, $S^{ \pm}$is the raising or lowering operator of the atom. $\Omega$ represents the strength of DDI between two atoms and $g$ is the coupling strength between the quantized cavity field and atoms. The DDI is defined in the form [2]

$$
J=\frac{3}{4}\left(\Gamma_{0} c^{3} / \omega_{a}^{3} r^{3}\right)\left(1-3 \cos ^{2} \varphi\right)
$$

where $r$ is the distance between the atoms and $\varphi$ is the angle between the interatomic axis and the atomic transition dipole moment. $\Gamma_{0}$ is the spontaneous emission rate in free space. Assuming that the direction of the polarized dipole moment of the atom is perpendicular to the interatomic axis. Thus, $\cos \varphi=0$, and the DDI intensity only depends on the distance of the two atoms in the cavity. In the presence of the DDI, it is convenient to study the dynamics of the atoms on the basis of collective states, so-called Dicke states, that are defined as $[2,34]$

$$
\begin{aligned}
& |e\rangle=\left|e_{1}\right\rangle \otimes\left|e_{2}\right\rangle \\
& |g\rangle=\left|g_{1}\right\rangle \otimes\left|g_{2}\right\rangle \\
& |s\rangle=\frac{1}{\sqrt{2}}\left(\left|e_{1}\right\rangle \otimes\left|g_{2}\right\rangle+\left|g_{1}\right\rangle \otimes\left|e_{2}\right\rangle\right) \\
& |a\rangle=\frac{1}{\sqrt{2}}\left(\left|e_{1}\right\rangle \otimes\left|g_{2}\right\rangle-\left|g_{1}\right\rangle \otimes\left|e_{2}\right\rangle\right)
\end{aligned}
$$

the corresponding eigenenergies are given by

$$
E_{e}=\omega_{a}, E_{g}=-\omega_{a}, E_{s}=\Omega, E_{a}=-\Omega .
$$

In Fig. 1, the degeneracy states $|s\rangle$ and $|a\rangle$ are degenerate in the absence of the DDI, where $E_{s}=E_{a}=0$. When the two atoms couple with DDI, i.e., $\Omega \neq 0$, the entangled states $|s\rangle$ and $|a\rangle$ are lifted in energy by $\Omega$ and $-\Omega$. Dissipation is induced by the interaction between the system and the environment. The atomic spontaneous decay $\gamma$, the collective decay among atoms $\gamma^{\prime}$ and the decay of the quantized cavity field $\kappa$ are considered. In the strong coupling regime, we assume $g>>\left(\kappa, \gamma, \gamma^{\prime}\right)$. In the BornMarkov approximation, the form of the master equation and the dissipation of the system can be expressed in the Lindblad form $[2,35]$

$$
\begin{aligned}
\dot{\rho}= & -i[H, \rho]+L_{\kappa} \rho+L_{\gamma} \rho+L_{\gamma^{\prime}} \rho \\
L_{\kappa} \rho= & \kappa\left(2 a \rho a^{\dagger}-a^{\dagger} a \rho-\rho a^{\dagger} a\right) \\
L_{\gamma} \rho= & \sum_{i=1}^{2} \gamma\left(2 S_{i}^{-} \rho S_{i}^{+}-S_{i}^{+} S_{i}^{-} \rho-\rho S_{i}^{+} S_{i}^{-}\right) \\
L_{\gamma^{\prime}} \rho= & \gamma^{\prime}\left(2 S_{2}^{-} \rho S_{1}^{+}-S_{1}^{+} S_{2}^{-} \rho-\rho S_{1}^{+} S_{2}^{-}\right) \\
& +\gamma^{\prime}\left(2 S_{1}^{-} \rho S_{2}^{+}-S_{2}^{+} S_{1}^{-} \rho-\rho S_{2}^{+} S_{1}^{-}\right) .
\end{aligned}
$$

Here, $L_{\kappa} \rho$ describes the coupling of the cavity field to the environment, and $L_{\gamma} \rho$ is the spontaneous emission of atoms. $\kappa$ and $\gamma$ are the corresponding decay rates. $L_{\gamma^{\prime}} \rho$ is the atomic collective spontaneous emission due to the DDI and $\gamma^{\prime}$ is the collective decay rate. The time evolution of the expectation values of the operator's for the atom-cavity system can be obtained with the master equation, (2.5) based on the Dicke states.

$$
\begin{aligned}
\langle\dot{a}\rangle= & \left(i \Delta_{c}-\kappa\right)\langle a\rangle-i g\left(\rho_{e s}+\rho_{s g}\right)-i \eta \\
\dot{\rho}_{e e}= & -4 \gamma \rho_{e e}-i \tilde{g}\left(\langle a\rangle \rho_{s e}-\left\langle a^{\dagger}\right\rangle \rho_{e s}\right) \\
\dot{\rho}_{s s}= & -2\left(\gamma+\gamma^{\prime}\right)\left(\rho_{s s}-\rho_{e e}\right) \\
& -i \tilde{g}\left(\left\langle a^{\dagger}\right\rangle \rho_{e s}-\langle a\rangle \rho_{s e}+\langle a\rangle \rho_{g s}-\left\langle a^{\dagger}\right\rangle \rho_{s g}\right) \\
\dot{\rho}_{a a}= & -2\left(\gamma-\gamma^{\prime}\right)\left(\rho_{a a}-\rho_{e e}\right) \\
\dot{\rho}_{g e}= & -2\left(i \Delta_{a}+\gamma\right) \rho_{g e}-i \tilde{g}\left\langle a^{\dagger}\right\rangle\left(\rho_{s e}-\rho_{g s}\right) \\
\dot{\rho}_{e a}= & -\left(3 \gamma-\gamma^{\prime}-i \Omega-i \Delta_{a}\right) \rho_{e a}-i g\langle a\rangle \rho_{s a} \\
\dot{\rho}_{g a}= & -\left(\gamma-\gamma^{\prime}+i \Omega+i \Delta_{a}\right) \rho_{g a}-i g\left\langle a^{\dagger}\right\rangle \rho_{s a}-2\left(\gamma-\gamma^{\prime}\right) \rho_{a e} \\
\dot{\rho}_{s a}= & -2(\gamma+i \Omega) \rho_{s a}-i g\left(\langle a\rangle \rho_{g a}+\left\langle a^{\dagger}\right\rangle \rho_{e a}\right) \\
\dot{\rho}_{e s}= & -e\left(3 \gamma+\gamma^{\prime}-i \Omega-i \Delta_{a}\right) \rho_{e s} \\
& -i \tilde{g}\left(\langle a\rangle \rho_{s s}-\langle a\rangle \rho_{e e}-\left\langle a^{\dagger}\right\rangle \rho_{e g}\right) \\
\dot{\rho}_{g s}= & i \tilde{g}\left\langle a^{\dagger}\right\rangle-\left(\gamma+\gamma^{\prime}-i \Omega+i \Delta_{a}\right) \rho_{g s}+2\left(\gamma+\gamma^{\prime}\right) \rho_{s e} \\
& -i \tilde{g}\left[\langle a\rangle\left(2 \rho_{s s}+\rho_{a a}+\rho_{e e}\right)-\left\langle a^{\dagger}\right\rangle \rho_{e g}\right]
\end{aligned}
$$

where $\tilde{g}=\sqrt{2} g$ and $\dot{\rho}_{g e}=\dot{\rho}_{e g}^{*}, \dot{\rho}_{e s}=\dot{\rho}_{s e}^{*}, \dot{\rho}_{g s}=\dot{\rho}_{s g}^{*}, \dot{\rho}_{a e}=$ $\dot{\rho}_{e a}^{*}, \dot{\rho}_{a g}=\dot{\rho}_{g a}^{*}, \dot{\rho}_{a s}=\dot{\rho}_{s a}^{*}$. In Eq. (2.6), a decorrelation between the operators of the atoms is used to classically explain the dynamics classically, and only mean value correlations of concern, i.e., correlations of the operators, are lost in this picture [36]. The equations of mo- 

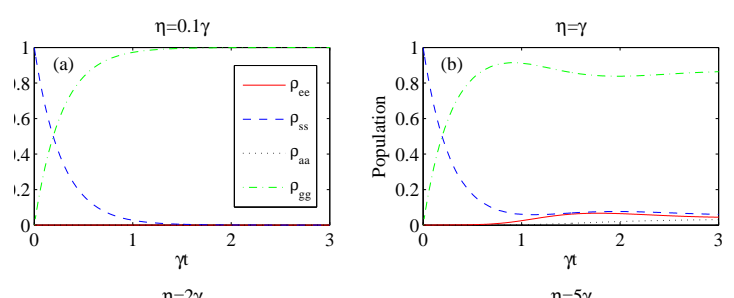

$\eta=2 \gamma$
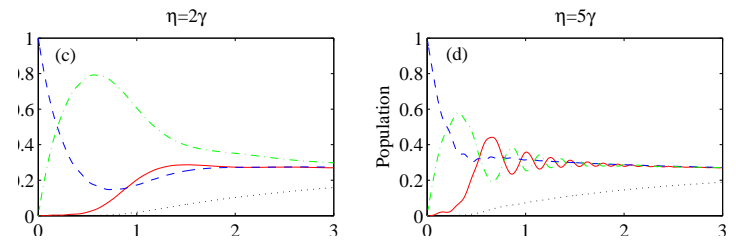

Figure 2: (Color online) Time evolution of population of Dicke state. Initially $\rho_{s s}=1$ and $\rho_{e e}=\rho_{a a}=\rho_{g g}=0$ with $\gamma^{\prime}=$ $0.8 \gamma, \Delta_{a}=\Delta_{c}, g=5 \gamma, \Omega=30 \gamma$ and $\kappa=0.5 \gamma$.

tion split into two independent sets, one composed of ten inhomogeneous equations and the other composed of six homogeneous coupled equations. The dynamic equations for the expectation values $\langle a\rangle$ given in Eqs.(2.6) demonstrate that the quantized cavity field can be changed by the coherence and the pump strength $\eta$. The effects of the quantized field will become weak With $\eta$ increases. Comparing the usual method to determine the coherence of the atom-cavity field system, characteristics demonstrating that the quantized cavity field is not a c-number function, but a changed one, can be identified. A relationship between cavity field operators and all the elements of the reduced density operator of the atoms occurs, leading to a nonlinear interplay between the quantized cavity field and the atoms.

The steady state behavior of level populations has been studied numerically for a wide range of parameter regimes when the driven field is considered to be classically [37] spanned by four product states $\left|e_{1}, e_{2}\right\rangle$, $\left|g_{1}, g_{2}\right\rangle,\left|e_{1}, g_{2}\right\rangle$, and $\left|g_{1}, e_{2}\right\rangle$. In what follows, a numerical method is used to study the evolution of the population and entanglement on the controlling parameters driven by quantized cavity field governed by the Dicke states according to Eq.(2.6).

\section{EVOLUTION OF POPULATION}

In this section, we will study the evolution of the atomic population using a numerical method, and we first consider the influence of pump strength $\eta$. In the presence of DDI between two atoms, the evolution of the population for different pump strengths $\eta$ is shown in Fig. 2 with atomic system in the symmetric state $|s\rangle$ initially. When the quantized cavity field is weakly pumped with $\eta=0.1 \gamma$, the state $|s\rangle$ rapidly decays to the ground state $|g\rangle$ without oscillation between other states because
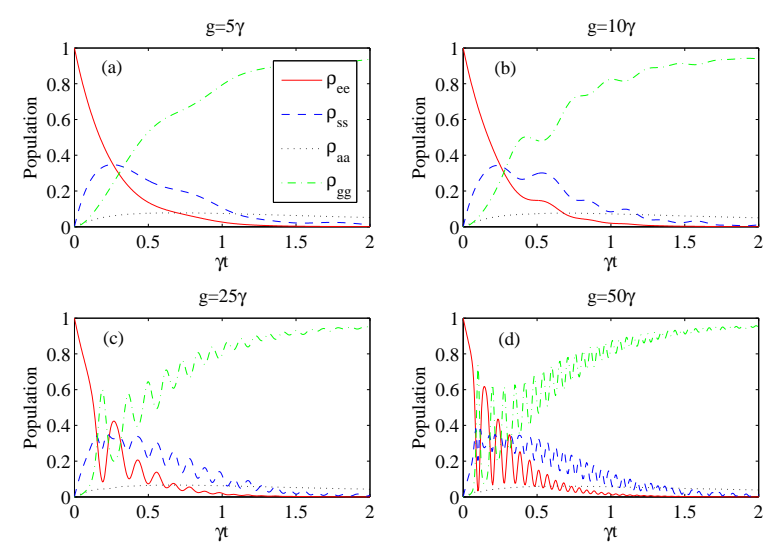

Figure 3: (Color online) Time evolution of population of Dicke state. Initially $\rho_{e e}=1$ and $\rho_{s s}=\rho_{a a}=\rho_{g g}=0$ with $\gamma^{\prime}=$ $0.8 \gamma, \Delta_{a}=\Delta_{c}=\Omega=0$ and $\kappa=\eta=0.5 \gamma$.

a weak pump field cannot provide sufficient photons for coupling among states. With the pump strength $\eta$ increasing, the number of photons can realize transitions between other states. Fig. 2(d) demonstrates that a two-photon transition process that is generated between states $|e\rangle$ and $|g\rangle$ in a strong pump regime. Here, the DDI shifts the energy level of $|s\rangle$, and this case finally leads to a non-transition process formed between states $|s\rangle$ and $|e\rangle$. Fig. 2(d) also demonstrates that the population of each state trends toward equivalence, that is, approximately 0.25 , which is similar to the results in a classical field case[30]. In addition, the two-photon transition process shown in Fig. 2 is more observable than those obtained the in classical field case.

Figure3 displays the dependence of population evolution on coupling strength $g$. We assume the atoms are in the excited state $|e\rangle$ initially, i.e., $\rho_{e e}=1$ without DDI and set $\Delta_{a}=0, \Delta_{c}=0$ first. It can be observed from Fig. 3(a) that the excited state decays to the intermediate state $|s\rangle$, which then decays to the ground state $|g\rangle$ with little oscillation when $g=5 \gamma$, which is because that photons can escape from the cavity before coupling [38]. With the increase in coupling strength $g$, the Rabi oscillation appears between the states $|e\rangle$ and $|s\rangle$. Moreover, the two-photon transition oscillation is also generated between the excited state $|e\rangle$ and ground state $|g\rangle$, which corresponds to the atomic transition process from state $|g\rangle$ directly absorbing two photons to the excited state $|e\rangle$ [21]. As $g$ increasing, the damped oscillation between states $|e\rangle$ and $|g\rangle$ appears.

In the presence of the DDI for parameters chosen in Fig. 3, numerical results show that though weak coupling among states $|e\rangle$ and $|s\rangle$ emerges, a weak twophoton transition process still exists. With increasing DDI strength, the coupling between states $|e\rangle$ and $|s\rangle$ is much suppressed, which is shown in Fig. 4, where atoms are initially the intermediate state $|s\rangle$. The population 


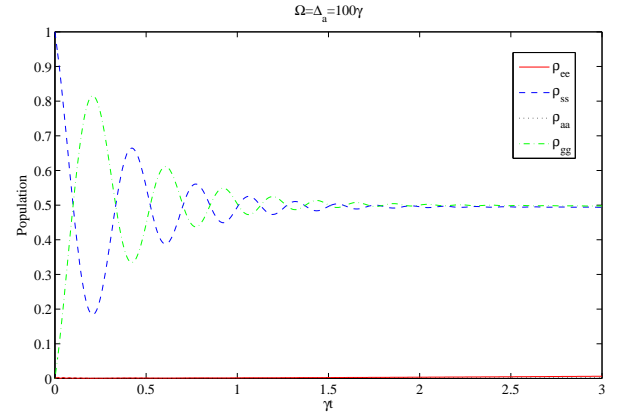

Figure 4: (Color online) Time evolution of population of Dicke state. Initially $\rho_{s s}=1$ and $\rho_{e e}=\rho_{a a}=\rho_{g g}=0$ with $\gamma^{\prime}=$ $0.8 \gamma, g=5 \gamma, \Delta_{c}=0, \kappa=0.5 \gamma, \eta=\gamma$ and $\Omega=\Delta_{a}=100 \gamma$.

of states $|e\rangle$ can be inhibited at all times, and the atomic system can eventually form a steady state. The large DDI and detuning lead to a resonance transition process occurring only between states $|s\rangle$ and $|g\rangle$. Therefore, one can use large DDI and accordingly change the detuning to enhance some transition processes between selected states and inhibit other processes.

Note that, the results here are different from those obtained in the classical field case [21]. In the classical field, the population of each states finally trends toward an equal value for strong coupling strength $g>>\left(\gamma, \gamma^{\prime}\right)$. This is because the quantum fluctuation effect caused by the quantized field enables photons to be easily absorbed by the vacuum state. The quantum fluctuation effect was more observable when the quantized cavity field was weakly pumped [30]. However, when the quantized cavity field was strongly pumped, the weak quantum fluctuation was overlapped by the strong pump field, and the quantized cavity field behaved as the classical field.

\section{MEASURE OF ENTANGLEMENT AND PHOTON COUNT RATE}

In this section, we use the widely applied concurrence to measure the entanglement between two atoms. The concurrence is defined as [39]

$$
\mathcal{C}=\max \left(0, \sqrt{\lambda_{1}}-\sqrt{\lambda_{2}}-\sqrt{\lambda_{3}}-\sqrt{\lambda_{4}}\right)
$$

where $\lambda_{i}$ is the eigenvalues of the matrix

$$
R=\rho \tilde{\rho}
$$

with

$$
\tilde{\rho}=\sigma_{y} \otimes \sigma_{y} \rho^{*} \sigma_{y} \otimes \sigma_{y},
$$
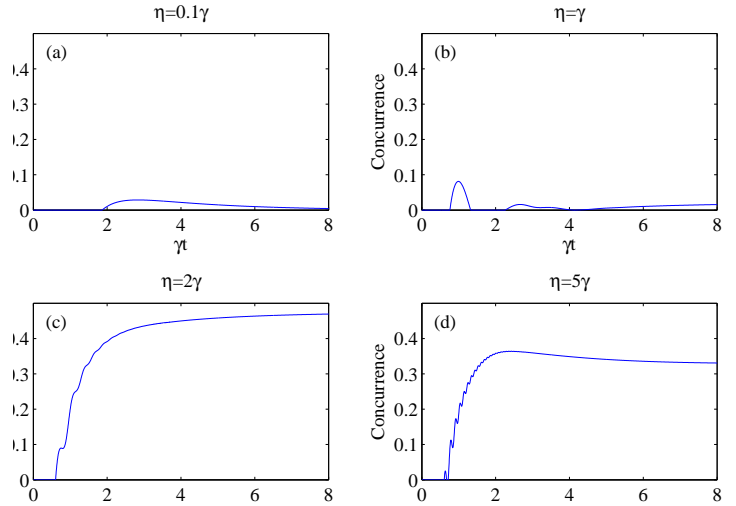

Figure 5: (Color online) Time evolution of concurrence. Initially $\rho_{e e}=1$ and $\rho_{s s}=\rho_{a a}=\rho_{g g}=0$ with $\gamma^{\prime}=0.8 \gamma, \Delta_{c}=$ $0, \kappa=0.5 \gamma, g=5 \gamma$ and $\Omega=\Delta_{a}=100 \gamma$.
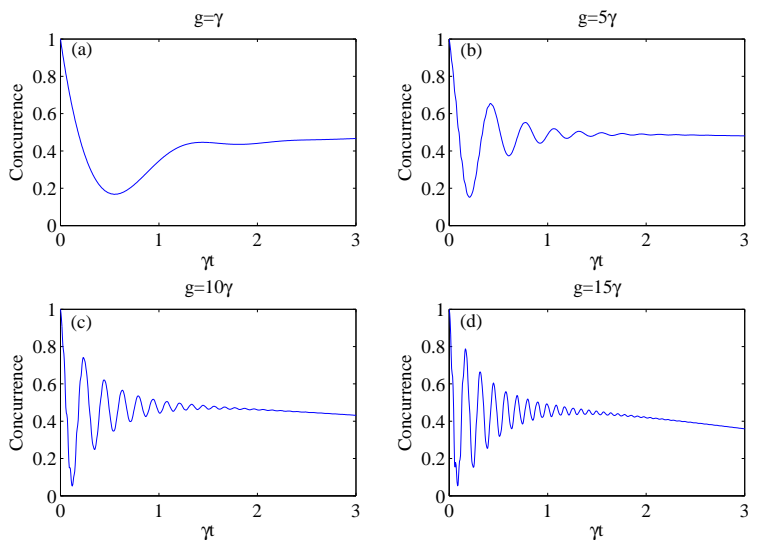

Figure 6: (Color online) Time evolution of concurrence. Initially $\rho_{e e}=1$ and $\rho_{s s}=\rho_{a a}=\rho_{g g}=0$ with $\gamma^{\prime}=0.8 \gamma, \Delta_{c}=$ $0, \kappa=0.5 \gamma, \eta=\gamma$ and $\Omega=\Delta_{a}=100 \gamma$.
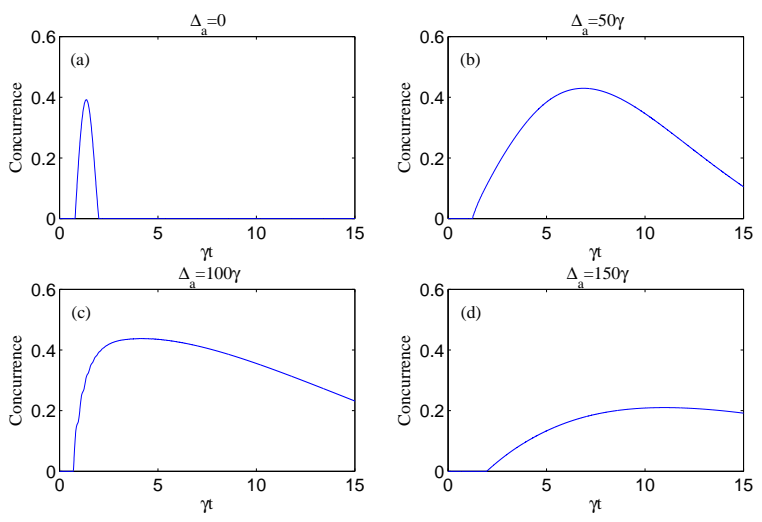

Figure 7: (Color online) Time evolution of concurrence. Initially $\rho_{e e}=1$ and $\rho_{s s}=\rho_{a a}=\rho_{g g}=0$ with $\gamma^{\prime}=0.8 \gamma, \Delta_{c}=$ $0, \kappa=0.5 \gamma, g=5 \gamma, \eta=\gamma$ and $\Omega=100 \gamma$. 
where $\sigma_{y}$ is Pauli matrix and $\rho^{*}$ is the complex conjugate of $\rho$. The system is entangled completely if $\mathcal{C}=1$ or independent while $\mathcal{C}=0$. The coherences between the triplet states and the antisymmetric state are zero for all times [21]. Then, we can simplify the density matrix of the atomic part of system denoted by

$$
\rho(t)=\left(\begin{array}{cccc}
\rho_{e e}(t) & \rho_{e s}(t) & 0 & \rho_{e g}(t) \\
\rho_{s e}(t) & \rho_{s s}(t) & 0 & \rho_{s g}(t) \\
0 & 0 & \rho_{a a}(t) & 0 \\
\rho_{g e}(t) & \rho_{g s}(t) & 0 & \rho_{g g}(t)
\end{array}\right)
$$

Now, we study the concurrence for different pump strengths $\eta$ with large DDI and detuning $\Delta_{a}=\Omega$, as displayed in Fig. 5. We can see that the concurrence was delayed as the system was prepared in state $|e\rangle$, and decayed to intermediate states $|s\rangle$ and $|a\rangle$ for a finite time, then decayed to ground state $|g\rangle$. When the system was weakly pumped, the entanglement was inhibited and diminished rapidly, which is because quantum fluctuation was pronounced than the weak pump field. When $\eta=2 \gamma$, the increased mean photon will induce the transition between states $|s\rangle$ and $|g\rangle$, then concurrence increased accordingly with the evolution of time. When $\eta=5 \gamma$, the quantized cavity field was strongly driven, as a similar explanation in the evolution of the population, and the evolution of entanglement was reduced to the classically driven case. However, the time of effective entanglement was different, which was decreased almost one order of magnitude due to quantum fluctuation.

The evolution of entanglement for different coupling strengths $g$ is plotted in Fig. 6. For weak coupling strength $g=\gamma$, the concurrence decreased rapidly from 1 to a steady value with a low frequency of oscillation. The evolution of concurrence overlapped completely with the evolution of state $|s\rangle$ population for the same given parameters. In other words, the concurrence was determined by the population of the state $|s\rangle$. The concurrence oscillations damped initially and then decayed slowly with increasing $g$. The reason the slow decay was due to the growth of population of $|s\rangle$ in a strong coupling regime.

In Fig. 7, the concurrence for different detunings $\Delta_{a}$ are shown. When $\Delta_{a}=0$, the concurrence had only one peak as a result of the two-photon transition between states $|e\rangle$ and $|g\rangle$. For large detunings compared with energy shifted by DDI, i.e., $\Delta_{a}=50 \gamma, 100 \gamma, 150 \gamma$, the concurrence displayed increases later, and the slight oscillation phenomenon appeared in Fig. 7(c) originating from the coupling between state $|s\rangle$ and $|g\rangle$.

Based on the coupled Eq.(2.6), the spectrum of the system can also be investigated. As is well known, observation of the normal mode splitting is a benchmark signature that a system has reached the strong coupling regime of cavity QED. We introduce the intracavity photon number $\left\langle a^{\dagger} a\right\rangle_{0}=\left|\langle a\rangle_{0}\right|^{2}$ to calculate the spectrum of the coupled atoms-cavity system. For this system, the modulus square of $\langle a\rangle_{0}$ can be calculated using the
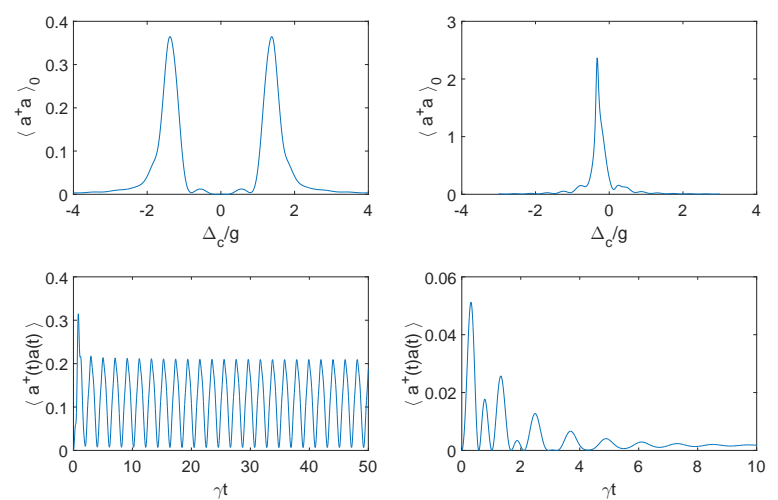

Figure 8: (Color online) The normal-mode spectrums and the photon count rate for different DDI intensities are shown. The parameters are (a) $\gamma=0.28 \mathrm{~g}, \gamma^{\prime}=0, \Omega=0, \Delta_{c}=\Delta_{a}, g=$ $1.0, \kappa=0.1 g, \eta=0.2 g$. (b) $\gamma=0.1 g, \gamma^{\prime}=0.1 g, \Omega=0, \Delta_{c}=$ $\Delta_{a}, g=1.0, \kappa=0.1 g, \eta=0.2 g$. (c) $\gamma=0.52, \gamma^{\prime}=0, \Omega=$ $5.77 \gamma, \Delta_{c}=\Delta_{a}=0, g=3.46 \gamma, \kappa=0.08 \gamma, \eta=0.96 \gamma$. (c) $\gamma=$ $0.52, \gamma^{\prime}=0, \Omega=0, \Delta_{c}=\Delta_{a}=0, g=3.46 \gamma, \kappa=0.08 \gamma, \eta=$ $0.96 \gamma$

steady-state solution of Eq.(2.6). Spectra which show the response of the intracavity photon number (proportional to the signal of a detector in transmission of the cavity) versus $\triangle_{c}$ are presented in Figs. 8( $\left.a\right)$ and $(b)$. It can be seen that, the spectrum shows the normal-mode splitting. When the DDI interaction is zero, i.e., $\Omega=0$, the amplitudes of both resonances are equal and their position are symmetric about $\Delta_{c}=0$. And the distance between the resonances reaches its minimum value of approximately $2 \sqrt{2} g$. However, with the DDI intensity increases, the system is approximately in the ground state $|g\rangle$, as shown in Fig.2(a). Then the atoms are not being excited, and a single peak is left and becomes higher and approaches $\Delta_{c}=0$, while the height of the right peak is greatly diminished and departs from $\Delta_{c}=0$.

The photon count rate $\left\langle a^{\dagger}(t) a(t)\right\rangle$ are plotted in Figs. $8(c)$ and $8(d)$ by solving Eq. (2.6) numerically. It is shown that the output photon shows a damped oscillation behaviour for small DDI. While for large DDI, an oscillation jumping between a high and a low value can be observed because of the constructive and destructive interfere of the scattered photons by the two atoms. This result is qualitatively consistent with the experimental data [16].

\section{CONCLUSION}

In this paper, we have studied the time evolution of population, the entanglement and the spectrum of two atoms placed in a quantized cavity field with DDI. It was found that the pump field played an important role in the system, and the result was different from those 
obtained in classical field. The nonlinear interaction between atoms and the quantized cavity will compare with the strength of pumped field. The population and entanglement show pronounced differences between weak and strong pump strength. The populations of some states can be enhanced due to large DDI and detunings.

\section{Acknowledgments}

This work was supported by NSFC under grants Nos. $11274148,11447221$.
[1] C. Cohen-Tannoudji, B. Diu and F. Laloë, 2005 Quantum Mechanics (Wiley-VCH) (1992).

[2] Z. Ficek, R. Tanaś, Phys. Rep. 372, 369 (2002).

[3] A. F. van Loo, A. Fedorov, K. Lalumière, B. C. Sanders, A. Blais, A. Wallraff, Science 342, 1494 (2013).

[4] P. Degenfeld-Schonburg, E. del Valle, and M. J. Hartmann, Phys. Rev. A. 85, 013842 (2012).

[5] J. R. Ott, M. Wubs, P. Lodahl, N. A. Mortensen,and R. Kaiser, Phys. Rev. A. 87, 061801 (2013).

[6] U. Eichmann, J. C. Bergquist, J. J. Bollinger, J. M. Gilligan, W. M. Itano, D. J. Wineland, and M. G. Raizen, Phys. Rev. Lett. 70, 2359 (1993).

[7] R. G. DeVoe and R. G. Brewer, Phys. Rev. Lett. 76, 2049 (1996).

[8] D. Riesch, K. Abich, W. Neuhauser, Ch. Wunderlich, and P.E. Toschek, Phys. Rev. A 65, 053401 (2002).

[9] A. Browaeys, D. Barredo and T. Lahaye, J. Phys. B: At. Mol. Opt. Phys. 49, 152001 (2016).

[10] S. Singh, C. H. Raymond Ooi, and Amrita, Phys. Rev. A. $86,023810(2012)$.

[11] H. M. Zou and M. F. Fang, Chin. Phys. B 25, 070305 (2016).

[12] Y. G. Huang, G. Chen, C. J. Jin, W. M. Liu, and X. H. Wang, Phys. Rev. A 85, 053827 (2012).

[13] Y. Q. Zhang, L. Tan, and P. Barker, Phys. Rev. A 89 043838 (2014)

[14] Y. M. Zheng, C. S. Hu, Z.B Yang, and H. Z. Wu, Chin. Phys. B 25, 104202 (2016).

[15] A. Neuzner, M. Körber, O. Morin, S. Ritter, and G. Rempe, Nat. Photon 10, 303 (2016).

[16] R. Reimann, W. Alt, T. Kampschulte, T. Macha, L. Ratschbacher, N. Thau, S. Yoon, and D. Meschede, Phys. Rev. Lett. 114, 023601 (2015).

[17] X. F. Zang, T. Zhou, B. Cai and Y. M. Zhu, J. Phys. B: At. Mol. Opt. Phys. 46, 145504(2013)

[18] X. F. Zang, T. Zhou, B. Cai, and Y. M. Zhu J. Opt. Soc. Am. B, 30, 1135(2013)

[19] K. M. Fan and G. F. Zhang, Eur. Phys. J. D 68,163 (2014).
[20] V. Petřinová, A. Lukř, J. Křepelka, and J. Peřina, Jr., Phys. Rev. A 90, 033428 (2014).

[21] K. Almutairi, R. Tanaś, and Z. Ficek, Phys. Rev. A 84, 013831 (2011).

[22] D. Kast and J. Ankerhold, Phys. Rev. B 90, 100301(R) (2014)

[23] E. K. Bashkirov, M.S. Mastyugin, Opt. Commun. 313, 170 (2014).

[24] J. Hu and H. Yu, 91, Phys. Rev. A 012327 (2015)

[25] M.R. Abbasi, Ann. Phys. 365, 198 (2016).

[26] O. de los Santos-Sánchez, C. González-Gutiérrez and J. Récamier, J. Phys. B: At. Mol. Opt. Phys. 49, 165503 (2016).

[27] M. Pleinert, J. von Zanthier, G. S. Agarwal, arXiv:1608.00137.

[28] P. W. H. Pinkse, T. Fischer, P. Maunz, G. Rempe, Nature 404, 365 (2000).

[29] C. Maschler, I.B. Mekhov, and H. Ritsch, Eur. Phys. J. D 46, 545 (2008).

[30] I. B Mekhov and H. Ritsch, J. Phys. B: At. Mol. Opt. Phys. 45, 102001(2012).

[31] H. Ritsch, P. Domokos, F. Brennecke, and T. Esslinger, Rev. Mod. Phys. 85, 553 (2013).

[32] L. Tan, L. W. Liu, Y. F Sun, Eur. Phys. J. D 61, 161 (2011).

[33] H. Wang, D. J. Goorskey and M. Xiao, Phys. Rev. A 65 011801

[34] R. H. Dicke, Phys. Rev. 93, 99 (1954).

[35] R. H. Lehmberg, Phys. Rev. A 2,883(1970).

[36] I. Schuster, A. Kubanek, A. Fuhrmanek, T. Puppe, P. W. H. Pinkse, K. Murr, and G. Rempe, Nat. Phys. 4, 382 (2008).

[37] P. A. Lakshmi, S. Ahmed, and A. Vudayagiri, arXiv: 1402.3777 .

[38] P. Lambropoulos, D. Petrosyan,Fundamentals of Quantum Optics and Quantum Information, Springer, Berlin, 2007.

[39] W. K. Wootters, Phys. Rev. Lett. 80, 2245(1998). 